\title{
ON BOOLEAN FUNCTIONS OF MANY VARIABLES*
}

\author{
BY \\ J. C. C. McKINSEY
}

1. Introduction. I here treat of some facts regarding Boolean functions of $n$ variables. The results are in part an extension to functions of $n$ variables of theorems already known for functions of one variable, in part a discussion of new problems.

By a function of $n$ Boolean variables I mean a rule whereby to each ordered set $x_{1}, \cdots, x_{n}$ of Boolean elements is assigned a Boolean element $z$. A function of Boolean variables is called a Boolean function if it can be expressed through a finite number of applications of the Boolean operations,$+ X$, and '. It is well known that every Boolean function $f\left(x_{1}, \cdots, x_{n}\right)$ can be expressed as $f(1, \cdots, 1) x_{1} \cdots x_{n}+\cdots+f(0, \cdots, 0) x_{1}^{\prime} \cdots x_{n}^{\prime}$. By a nonBoolean function, I mean a function of Boolean variables which is not a Boolean function. I shall be concerned mainly, as already mentioned, with Boolean functions, but an occasional reference will be made to non-Boolean functions.

In \$\$2-6 I discuss "monotone" Boolean functions, which are analogous to the monotone functions of ordinary analysis. I establish conditions that functions be monotone non-decreasing and monotone non-increasing. In $\$ \S 7-9$ I discuss a more special kind of functions, namely, "additive" and "subtractive" functions. In $\$ \$ 10$ and 11 I define and discuss an analogue, for Boolean functions of the "continuity" of classical analysis. I show here that all Boolean functions are "continuous" and suggest the importance of continuity with regard to non-Boolean functions. In $\$ 12$ I discuss inverse functions, finding all the domains within which a function of $n$ variables has a one-valued inverse.

Schmidt $\dagger$ obtained some interesting facts concerning Boolean functions of one variable. Schmidt's results are included in my Theorems 15, 17, 20, 24, and 30 . Theorems $15,17,20$, and 24 constitute a generalization to functions of $n$ variables of his "Principal Theorem" $\ddagger$ concerning Boolean functions of one variable. In $\$ \$ 5$ and 8 I make some remarks on the ways in which this

* Presented to the Society, April 11, 1936; received by the editors February 28, 1936.

$\dagger$ Karl Schmidt, The theory of functions of one Boolean variable, these Transactions, vol. 23 (1922), pp. 212-222. All later references to Schmidt are to this paper.

$\ddagger$ The theorem is as follows: Every change of $x$, or part of $x$, in the increment region $a b^{\prime}$ produces the same change in $a x+b x^{\prime}$; in the decrement region $a^{\prime} b$ it produces the opposite effect; and a change of $x$ in any other region has no effect whatsoever on $a x+b x^{\prime}$, which always occupies ab and never enters $a^{\prime} b^{\prime}$. 
theorem fails to admit of generalization. Theorem 30 gives the domains within which a function of $n$ variables has a one-valued inverse; this problem was only partially solved by Schmidt for functions of one variable.

Throughout the paper I use, besides the familiar Boolean operations, + , $X$, and ', the operations $a \circ b$ and $a \Delta b$, which have been recently discussed by Bernstein.* These operations are defined as follows: $a \circ b=a^{\prime} b+a b^{\prime}$, $a \Delta b=a b+a^{\prime} b^{\prime}$. From their definitions it is easily seen that both operations enjoy the commutative and associative properties and that $a \Delta b=(a \circ b)^{\prime}$. Other properties will be found in Bernstein's paper.

2. Monotone non-decreasing functions. One of the important general types of functions of a real variable is the set of monotone functions. A function of a real variable is monotone non-decreasing if it does not decrease when the argument increases, and monotone non-increasing if it does not increase when the argument increases. Thus, for example, the function $f(x)=\sin x$ is monotone non-decreasing in the interval $(0, \pi / 2)$ and monotone non-increasing in the interval $(\pi / 2, \pi)$. This classification rests upon the use of the relation $<$ as holding between real numbers, and hence cannot be applied directly to functions of Boolean variables. If we consider one of the most important interpretations of Boolean algebra, however, namely, the interpretation of the algebra as a calculus of classes; we are led to consider a function as monotone non-decreasing when it has the following characteristic: If the argument changes from one class to a more inclusive class, the function changes from one class to a more inclusive class. And analogously for monotone non-increasing functions. These notions are made more precise by the following definitions.

Definition 1. A Boolean function $f\left(x_{1}, \cdots, x_{n}\right)$ is said to be monotone non-decreasing with respect to $x_{1}$ in the domain $(A, B)$ if, for all $\alpha$ in $(A, B)$,

$$
f\left(x_{1}, \cdots, x_{n}\right)<f\left(x_{1}+\alpha, x_{2}, \cdots, x_{n}\right)
$$

independent of the choice of $x_{1}, \cdots, x_{n}$. If $f\left(x_{1}, \cdots, x_{n}\right)$ is monotone nondecreasing with respect to $x_{1}$ in the domain $(0,1)$, we say that $f\left(x_{1}, \cdots, x_{n}\right)$ is monotone non-decreasing with respect to $x_{1}$ everywhere.

Definition 2. A Boolean function $f\left(x_{1}, \cdots, x_{n}\right)$ is said to be monotone non-increasing with respect to $x_{1}$ in the domain $(A, B)$ if, for all $\alpha$ in $(A, B)$,

$$
f\left(x_{1}+\alpha, x_{2}, \cdots, x_{n}\right)<f\left(x_{1}, \cdots, x_{n}\right)
$$

independent of the choice of $x_{1}, \cdots, x_{n}$. If $f\left(x_{1}, \cdots, x_{n}\right)$ is monotone non-

* B. A. Bernstein, Postulates for Boolean algebra involving the operation of complete disjunction, to appear in the Annals of Mathematics. I take this opportunity of acknowledging my indebtedness to Professor Bernstein for his valuable suggestions regarding the present paper. 
increasing with respect to $x_{1}$ in the domain $(0,1)$, we say that $f\left(x_{1}, \cdots, x_{n}\right)$ is monotone non-increasing with respect to $x_{1}$ everywhere.

Thus, for example, the Boolean function of one variable $f(x)=a x$ is monotone non-decreasing everywhere; for,

$$
f(x)=a x<a x+a \alpha=a(x+\alpha)=f(x+\alpha)
$$

for any $x$. Similarly the function $f(x)=a x^{\prime}$ is monotone non-increasing everywhere; for,

$$
f(x+\alpha)=a(x+\alpha)^{\prime}=a x^{\prime} \alpha^{\prime}<a x^{\prime}=f(x)
$$

for any $x$.

I now find a condition that a function of one variable be monotone nondecreasing in a domain.

THEOREM 1. A necessary and sufficient condition that a given function $f(x)$ of one variable be monotone non-decreasing in the domain $(A, B)$ is that

$$
B<f(1)+f^{\prime}(0) \text {. }
$$

Proof. To say that $f(x)$ is monotone non-decreasing in $(A, B)$ is, by definition, equivalent to saying that for all $\alpha$ in $(A, B)$ we have

$$
f(x)<f(x+\alpha) \text {. }
$$

But (1) is equivalent to

$$
f(x) f^{\prime}(x+\alpha)=0
$$

which is equivalent to

$$
f(0) f^{\prime}(1) \alpha x^{\prime}=0 .
$$

Since (3) is an identity in $x$, it is equivalent to

$$
f(0) f^{\prime}(1) \alpha=0,
$$

or to

$$
\alpha<f(1)+f^{\prime}(0) .
$$

If (5) holds for all $\alpha$ in $(A, B)$, it holds in particular for $\alpha=B$, since $B$ is in $(A, B)$; moreover, if (5) holds for $B$, it holds for all $\alpha$ in $(A, B)$, since if $\alpha$ is in $(A, B)$ we have $\alpha<B$. Hence to say that (5) holds for all $\alpha$ in $(A, B)$ is equivalent to saying that

$$
B<f(1)+f^{\prime}(0)
$$

as was to be shown. 
I next generalize Theorem 1 to functions of $n$ variables.

THEOREM 2. A necessary and sufficient condition that a given function $f\left(x_{1}, \cdots, x_{n}\right)$ be monotone non-decreasing with respect to $x_{1}$ in the domain $(A, B)$ is that

$$
B<\prod_{\alpha_{i}=0,1}\left[f\left(1, \alpha_{2}, \cdots, \alpha_{n}\right)+f^{\prime}\left(0, \alpha_{2}, \cdots, \alpha_{n}\right)\right] .
$$

Proof. I prove the theorem by mathematical induction on $n$.

For a function of one variable, we have Theorem 1.

Suppose, now, that the theorem is true for $n=k$. Let $f\left(x_{1}, \cdots, x_{k+1}\right)$ be any function of $k+1$ variables. For any fixed value, $z$, of $x_{k+1}, f\left(x_{1}, \cdots, x_{k}, z\right)$ is a function of $k$ variables. Then, since by hypothesis the theorem holds for a function of $k$ variables, we have: A necessary and sufficient condition that $f\left(x_{1}, \cdots, x_{k}, z\right)$ be monotone non-decreasing with respect to $x_{1}$ for the domain $(A, B)$ is that

$$
B<\prod_{\alpha_{i}=0,1}\left[f\left(1, \alpha_{2}, \cdots, \alpha_{k}, z\right)+f^{\prime}\left(0, \alpha_{2}, \cdots, \alpha_{k}, z\right)\right] .
$$

Since (1) is the required necessary and sufficient condition for each $z$, we may regard $z$ as a variable in the condition. Thus, a necessary and sufficient condition that $f\left(x_{1}, \cdots, x_{n}\right)$ be monotone non-decreasing in $(A, B)$ is that (1) hold for all $z$. But to say that (1) holds for all $z$ is equivalent to saying

$$
\left\{\begin{array}{l}
B<\prod_{\alpha_{i}=0,1}\left[f\left(1, \alpha_{2}, \cdots, \alpha_{k}, 1\right)+f^{\prime}\left(0, \alpha_{2}, \cdots, \alpha_{k}, 1\right)\right] \\
B<\prod_{\alpha_{i}=0,1}\left[f\left(1, \alpha_{2}, \cdots, \alpha_{k}, 0\right)+f^{\prime}\left(0, \alpha_{2}, \cdots, \alpha_{k}, 0\right)\right] .
\end{array}\right.
$$

And conditions (2) are equivalent to the condition specified in the theorem. Hence the theorem is proved.

THEOREM 3. A necessary and sifficient condition that a function $f\left(x_{1}, \cdots, x_{n}\right)$ be monotone non-decreasing with respect to $x_{1}$ everywhere is that

$$
f\left(0, \alpha_{2}, \cdots, \alpha_{n}\right)<f\left(1, \alpha_{2}, \cdots, \alpha_{n}\right) \quad\left[\alpha_{i}=0,1\right] .
$$

Proof. For, to say $f\left(x_{1}, \cdots, x_{n}\right)$ is monotone non-decreasing with respect to $x_{1}$ everywhere is, by definition, to say that $f\left(x_{1}, \cdots, x_{n}\right)$ is monotone nondecreasing with respect to $x_{1}$ in the domain $(0,1)$. This, by Theorem 2 , is equivalent to

or to

$$
\prod_{\alpha_{i}=0,1}\left[f\left(1, \alpha_{2}, \cdots, \alpha_{n}\right)+f^{\prime}\left(0, \alpha_{2}, \cdots, \alpha_{n}\right)\right]=1,
$$




$$
f\left(1, \alpha_{2}, \cdots, \alpha_{n}\right)+f^{\prime}\left(0, \alpha_{2}, \cdots, \alpha_{n}\right)=1 \quad\left[\alpha_{i}=0,1\right] .
$$

The last equations are equivalent to the given inclusions.

THEOREM 4. A necessary and sufficient condition that a function $f\left(x_{1}, \cdots, x_{n}\right)$ be monotone non-decreasing with respect to $x_{1}$ everywhere is that $f\left(x_{1}, \cdots, x_{n}\right)$ be expressible in a form which does not involve $x_{1}^{\prime}$ (but which may involve $x_{1}$ ).

Proof. If $f\left(x_{1}, \cdots, x_{n}\right)$ is monotone non-decreasing with respect to $x_{1}$ everywhere, then, by the preceding theorem, we have

$$
f\left(0, \alpha_{2}, \cdots, \alpha_{n}\right)<f\left(1, \alpha_{2}, \cdots, \alpha_{n}\right) \quad\left[\alpha_{i}=0,1\right] .
$$

Hence

$$
f\left(0, \alpha_{2}, \cdots, \alpha_{n}\right)=f\left(0, \alpha_{2}, \cdots, \alpha_{n}\right) f\left(1, \alpha_{2}, \cdots, \alpha_{n}\right) \quad\left[\alpha_{i}=0,1\right] .
$$

Thus

$$
\begin{aligned}
f\left(x_{1}, \cdots, x_{n}\right)= & f(1, \cdots, 1) x_{1} \cdots x_{n}+\cdots+f(1,0, \cdots, 0) x_{1} x_{2}^{\prime} \cdots x_{n}^{\prime} \\
& +f(0,1, \cdots, 1) f(1, \cdots, 1) x_{1}^{\prime} x_{2} \cdots x_{n}+\cdots \\
& +f(0, \cdots, 0) f(1,0, \cdots, 0) x_{1}^{\prime} \cdots x_{n}^{\prime} \\
= & f(1, \cdots, 1)\left[x_{1}+x_{1}^{\prime} f(0,1, \cdots, 1)\right] x_{2} \cdots x_{n}+\cdots \\
& +f(1,0, \cdots, 0)\left[x_{1}+x_{1}^{\prime} f(0, \cdots, 0)\right] x_{2}^{\prime} \cdots x_{n}^{\prime} \\
= & f(1, \cdots, 1)\left[x_{1}+f(0,1, \cdots, 1)\right] x_{2} \cdots x_{n}+\cdots \\
& +f(1,0, \cdots, 0)\left[x_{1}+f(0, \cdots, 0)\right] x_{2}^{\prime} \cdots x_{n}^{\prime}
\end{aligned}
$$

which is a form not involving $x_{1}^{\prime}$. Hence, the condition is necessary.

Suppose, on the other hand, that $f\left(x_{1}, \cdots, x_{n}\right)$ can be expressed in a form not involving $x_{1}^{\prime}$. The most general such form is

$$
f\left(x_{1}, \cdots, x_{n}\right)=x_{1} g\left(x_{2}, \cdots, x_{n}\right)+h\left(x_{2}, \cdots, x_{n}\right) .
$$

Then we have

$$
\begin{aligned}
& f\left(0, \alpha_{2}, \cdots, \alpha_{n}\right)=h\left(\alpha_{2}, \cdots, \alpha_{n}\right), \\
& f\left(1, \alpha_{2}, \cdots, \alpha_{n}\right)=g\left(\alpha_{2}, \cdots, \alpha_{n}\right)+h\left(\alpha_{2}, \cdots, \alpha_{n}\right) .
\end{aligned}
$$

Therefore,

$$
f\left(0, \alpha_{2}, \cdots, \alpha_{n}\right)<f\left(1, \alpha_{2}, \cdots, \alpha_{n}\right) \quad\left[\alpha_{i}=0,1\right],
$$

and hence, again by the preceding theorem, $f\left(x_{1}, \cdots, x_{n}\right)$ is monotone nondecreasing with respect to $x_{1}$ everywhere. Hence, the condition is sufficient.

THEOREM 5. A necessary and sufficient condition that $f\left(x_{1}, \cdots, x_{n}\right)$ be monotone non-decreasing with respect to $x_{1}$ everywhere is that, for every $u$ and $v$,

$$
f\left(u+v, x_{2}, \cdots, x_{n}\right)=f\left(u, x_{2}, \cdots, x_{n}\right)+f\left(v, x_{2}, \cdots, x_{n}\right) .
$$


Proof. To say that

$$
f\left(u+v, x_{2}, \cdots, x_{n}\right)=f\left(u, x_{2}, \cdots, x_{n}\right)+f\left(v, x_{2}, \cdots, x_{n}\right)
$$

holds for all $u$ and $v$, is equivalent to saying it holds for $u, v=0,1$. But (1) becomes an identity when we substitute $u=v=1$, and likewise when we substitute $u=v=0$. Furthermore, we obtain the same condition when we put $u=1, v=0$ as when we put $u=0, v=1$. Hence to say that (1) holds for all $u$ and $v$ is equivalent to saying that

$$
f\left(1, x_{2}, \cdots, x_{n}\right)=f\left(1, x_{2}, \cdots, x_{n}\right)+f\left(0, x_{2}, \cdots, x_{n}\right),
$$

which is in turn equivalent to

$$
f\left(0, x_{2}, \cdots, x_{n}\right)<f\left(1, x_{2}, \cdots, x_{n}\right) .
$$

Since (3) is an identity in $x_{2}, \cdots, x_{n}$ it is the same as the condition, given in Theorem 3 , that $f\left(x_{1}, \cdots, x_{n}\right)$ be monotone non-decreasing with respect to $x_{1}$ everywhere. Hence, (1) is a necessary and sufficient condition that $f\left(x_{1}, \cdots, x_{n}\right)$ be monotone non-decreasing with respect to $x_{1}$ everywhere.

The following theorem is proved in a similar way.

THEOREM 6. A necessary and sufficient condition that $f\left(x_{1}, \cdots, x_{n}\right)$ be monotone non-decreasing with respect to $x_{1}$ everywhere is that, for every $u$ and $v$

$$
f\left(u v, x_{2}, \cdots, x_{n}\right)=f\left(u, x_{2}, \cdots, x_{n}\right) f\left(v, x_{2}, \cdots, x_{n}\right) .
$$

The following theorem gives a condition for monotone non-decreasing domains which might have been taken as the definition. Definition 1 was selected only because its content is slightly more obvious intuitively

THEOREM 7. A necessary and sufficient condition that a function $f\left(x_{1}, \cdots, x_{n}\right)$ be monotone non-decreasing with respect to $x_{1}$ in the domain $(A, B)$ is that, for all $\alpha$ in $(A, B)$, we have

$$
f\left(x_{1} \alpha^{\prime}, x_{2}, \cdots, x_{n}\right)<f\left(x_{1}, x_{2}, \cdots, x_{n}\right)
$$

independent of the choice of $x_{1}, \cdots, x_{n}$.

Proof. Let $f(x)$ be a function of one variable which satisfies

$$
f\left(x \alpha^{\prime}\right)<f(x)
$$

identically for all $\alpha$ in $(A, B)$. Condition (1) reduces to

$$
f^{\prime}(1) f(0) \alpha x=0 .
$$

Since (2) is an identity in $x$, it is equivalent to

$$
f^{\prime}(1) f(0) \alpha=0,
$$


which is equivalent to

$$
\alpha<f(1)+f^{\prime}(0)
$$

Thus,

$$
B<f(1)+f^{\prime}(0) .
$$

It may now be shown, by mathematical induction on $n$, that $f\left(x_{1}, \cdots, x_{n}\right)$ satisfies

$$
f\left(x_{1} \alpha^{\prime}, x_{2}, \cdots, x_{n}\right)<f\left(x_{1}, \cdots, x_{n}\right)
$$

identically for all $\alpha$ in $(A, B)$ if and only if

$$
B<\prod_{\alpha_{2}=0,1}\left[f\left(1, \alpha_{2}, \cdots, \alpha_{n}\right)+f^{\prime}\left(0, \alpha_{2}, \cdots, \alpha_{n}\right)\right] .
$$

But this is the condition, found in Theorem 2, that $f\left(x_{1}, \cdots, x_{n}\right)$ be monotone non-decreasing in $(A, B)$.

3. Monotone non-increasing functions. The proofs of the following seven theorems are omitted, since they are closely analogous to the proofs of the corresponding theorems on monotone non-decreasing functions.

THEOREM 8. A necessary and sufficient condition that a given function $f(x)$ of one variable be monotone non-increasing in the domain $(A, B)$ is that

$$
B<f^{\prime}(1)+f(0) .
$$

THEOREM 9. A necessary and sufficient condition that a given function $f\left(x_{1}, \cdots, x_{n}\right)$ be monotone non-increasing with respect to $x_{1}$ in the domain $(A, B)$ is that

$$
B<\prod_{\alpha_{i}=0,1}\left[f^{\prime}\left(1, \alpha_{2}, \cdots, \alpha_{n}\right)+f\left(0, \alpha_{2}, \cdots, \alpha_{n}\right)\right] .
$$

THEOREM 10. A necessary and sufficient condition that a function $f\left(x_{1}, \cdots, x_{n}\right)$ be monotone non-increasing with respect to $x_{1}$ everywhere is that

$$
f\left(1, \alpha_{2}, \cdots, \alpha_{n}\right)<f\left(0, \alpha_{2}, \cdots, \alpha_{n}\right) \quad\left[\alpha_{i}=0,1\right] .
$$

THEOREM 11. A necessary and sufficient condition that a function $f\left(x_{1}, \cdots, x_{n}\right)$ be monotone non-increasing with respect to $x_{1}$ everywhere is that $f\left(x_{1}, \cdots, x_{n}\right)$ be expressible in a form which does not involve $x_{1}$ (but which may involve $\left.x_{1}^{\prime}\right)$.

TheORem 12. A necessary and sufficient condition that $f\left(x_{1}, \cdots, x_{n}\right)$ be monotone non-increasing with respect to $x_{1}$ everywhere is that, for every $u$ and $v$,

$$
f\left(u+v, x_{2}, \cdots, x_{n}\right)=f\left(u, x_{2}, \cdots, x_{n}\right) f\left(v, x_{2}, \cdots, x_{n}\right) .
$$


THEOREM 13. A necessary and sufficient condition that $f\left(x_{1}, \cdots, x_{n}\right)$ be monotone non-increasing with respect to $x_{1}$ everywhere is that, for every $u$ and $v$,

$$
f\left(u v, x_{2}, \cdots, x_{n}\right)=f\left(u, x_{2}, \cdots, x_{n}\right)+f\left(v, x_{2}, \cdots, x_{n}\right) .
$$

THEOREM 14. A necessary and sufficient condition that a function $f\left(x_{1}, \cdots, x_{n}\right)$ be monotone non-increasing with respect to $x_{1}$ in the domain $(A, B)$ is that, for all $\alpha$ in $(A, B)$, we have

$$
f\left(x_{1}, x_{2}, \cdots, x_{n}\right)<f\left(x_{1} \alpha^{\prime}, x_{2}, \cdots, x_{n}\right)
$$

independent of the choice of $x_{1}, \cdots, x_{n}$.

4. Functions both monotone non-decreasing and monotone non-increasing. Constant functions. We prove the following theorem.

THEOREM 15. A necessary and sufficient condition that a function $f\left(x_{1}, \cdots, x_{n}\right)$ be both monotone non-decreasing and monotone non-increasing with respect to $x_{1}$ in the domain $(A, B)$ is that

$$
B<\prod_{\alpha_{i}=0,1}\left[f\left(1, \alpha_{2}, \cdots, \alpha_{n}\right) \Delta f\left(0, \alpha_{2}, \cdots, \alpha_{n}\right)\right] .
$$

Proof. To say that $f\left(x_{1}, \cdots, x_{n}\right)$ is both monotone non-decreasing and monotone non-increasing with respect to $x_{1}$ in $(A, B)$ is, by Theorems 2 and 9 , equivalent to saying that

$$
\left\{\begin{array}{l}
B<\prod_{\alpha_{i}=0,1}\left[f\left(1, \alpha_{2}, \cdots, \alpha_{n}\right)+f^{\prime}\left(0, \alpha_{2}, \cdots, \alpha_{n}\right)\right], \\
B<\prod_{\alpha_{i}=0,1}\left[f^{\prime}\left(1, \alpha_{2}, \cdots, \alpha_{n}\right)+f\left(0, \alpha_{2}, \cdots, \alpha_{n}\right)\right] .
\end{array}\right.
$$

Conditions (1) are equivalent to the single condition

$$
\begin{array}{r}
B<\prod_{\alpha_{i}=0,1}\left[f\left(1, \alpha_{2}, \cdots, \alpha_{n}\right)+f^{\prime}\left(0, \alpha_{2}, \cdots, \alpha_{n}\right)\right] \\
\prod_{\alpha_{i}=0,1}\left[f^{\prime}\left(1, \alpha_{2}, \cdots, \alpha_{n}\right)+f\left(0, \alpha_{2}, \cdots, \alpha_{n}\right)\right],
\end{array}
$$

or

$$
\begin{array}{r}
B<\prod_{\alpha_{i}=0,1}\left[f\left(1, \alpha_{2}, \cdots, \alpha_{n}\right)+f^{\prime}\left(0, \alpha_{2}, \cdots, \alpha_{n}\right)\right] \\
\cdot\left[f^{\prime}\left(1, \alpha_{2}, \cdots, \alpha_{n}\right)+f\left(0, \alpha_{2}, \cdots, \alpha_{n}\right)\right],
\end{array}
$$

or, finally,

$$
B<\prod_{\alpha_{i}=0,1}\left[f\left(1, \alpha_{2}, \cdots, \alpha_{n}\right) \Delta f\left(0, \alpha_{2}, \cdots, \alpha_{n}\right)\right]
$$


THEOREM 16. A necessary and sufficient condition that $f\left(x_{1}, \cdots, x_{n}\right)$ be both monotone non-decreasing and monotone non-increasing with respect to $x_{1}$ everywhere is that

$$
f\left(1, \alpha_{2}, \cdots, \alpha_{n}\right)=f\left(0, \alpha_{2}, \cdots, \alpha_{n}\right) \quad\left[\alpha_{i}=0,1\right],
$$

hence, that $f\left(x_{1}, \cdots, x_{n}\right)$ be expressible in a form which involves neither $x_{1}$ nor $x_{1}^{\prime}$.

Proof. The theorem is an immediate consequence of Theorem 15.

By saying that $f\left(x_{1}, \cdots, x_{n}\right)$ is constant with respect to $x_{1}$ in the domain $(A, B)$, I mean that $f\left(x_{1}, \cdots, x_{n}\right)$ is unchanged when $x_{1}$ assumes or loses any increment from $(A, B)$.

THEOREM 17. A necessary and sufficient condition that $f\left(x_{1}, \cdots, x_{n}\right)$ be constant with respect to $x_{1}$ in a domain $(A, B)$ is that $f\left(x_{1}, \cdots, x_{n}\right)$ be both monotone non-decreasing and monotone non-increasing with respect to $x_{1}$ in the domain $(A, B)$.

Proof. To say that $f\left(x_{1}, \cdots, x_{n}\right)$ is constant with respect to $x_{1}$ in the domain $(A, B)$ is to say that for all $\alpha$ in $(A, B)$

$$
\begin{aligned}
f\left(x_{1}+\alpha, x_{2}, \cdots, x_{n}\right) & =f\left(x_{1}, \cdots, x_{n}\right), \\
f\left(x_{1} \alpha^{\prime}, x_{2}, \cdots, x_{n}\right) & =f\left(x_{1}, \cdots, x_{n}\right) .
\end{aligned}
$$

Conditions (1) are equivalent to the four conditions

$$
\left\{\begin{aligned}
f\left(x_{1}+\alpha, x_{2}, \cdots, x_{n}\right) & <f\left(x_{1}, \cdots, x_{n}\right), \\
f\left(x_{1}+\alpha, x_{2}, \cdots, x_{n}\right) & >f\left(x_{1}, \cdots, x_{n}\right), \\
f\left(x_{1} \alpha^{\prime}, x_{2}, \cdots, x_{n}\right) & <f\left(x_{1}, \cdots, x_{n}\right), \\
f\left(x_{1} \alpha^{\prime}, x_{2}, \cdots, x_{n}\right) & >f\left(x_{1}, \cdots, x_{n}\right) .
\end{aligned}\right.
$$

By Theorems 7 and 14, respectively, the third and fourth conditions of (2) are consequences of the first and second. Hence conditions (1) are equivalent to

$$
\begin{aligned}
& f\left(x_{1}+\alpha, x_{2}, \cdots, x_{n}\right)<f\left(x_{1}, \cdots, x_{n}\right), \\
& f\left(x_{1}+\alpha, x_{2}, \cdots, x_{n}\right)>f\left(x_{1}, \cdots, x_{n}\right) .
\end{aligned}
$$

Hence the theorem is established.

THEOREM 18. A necessary and sufficient condition that $f\left(x_{1}, \cdots, x_{n}\right)$ be constant with respect to $x_{1}$ everywhere is that

$$
f\left(1, \alpha_{2}, \cdots, \alpha_{n}\right)=f\left(0, \alpha_{2}, \cdots, \alpha_{n}\right) \quad\left[\alpha_{i}=0,1\right],
$$

hence, that $f\left(x_{1}, \cdots, x_{n}\right)$ be expressible in a form which involves neither $x_{1}$ nor $x_{1}^{\prime}$. 
Proof. The theorem is an immediate consequence of Theorems 16 and 17.

5. Remarks on the variation of functions. It is of some interest to notice that the theorems given so far are sufficient to give a complete, though rough, characteriziation of the variation of a function of one variable. When we are given the function $f(x)=a x+b x^{\prime}$, then we may consider the universe as falling into the four regions: $a b, a b^{\prime}, a^{\prime} b, a^{\prime} b^{\prime}$. From Theorems $15,17,1,7,8$, and 14 we now see that $f(x)$ is affected as follows when $x$ assumes or loses increments from these regions:

region if $x$ assumes an increment

$a b \quad f(x)$ is unchanged

$a^{\prime} b^{\prime} \quad f(x)$ is unchanged

$a b^{\prime} \quad f(x)$ assumes an increment*

$a^{\prime} b \quad f(x)$ loses an increment if $x$ loses an increment

$f(x)$ is unchanged

$f(x)$ is unchanged

$f(x)$ loses an increment

$f(x)$ assumes an increment

For functions of more than one variable, however, the theorems are not sufficient to allow such a complete characterization. For a function of two variables, for example, $f(x, y)=a x y+b x y^{\prime}+c x^{\prime} y+d x^{\prime} y^{\prime}$, we may consider the universe as falling into sixteen regions:

$\begin{array}{llll}a b c d, & a b c^{\prime} d, & a^{\prime} b^{\prime} c d, & a^{\prime} b c d^{\prime}, \\ a b^{\prime} c d^{\prime}, & a b c d^{\prime}, & a^{\prime} b^{\prime} c d^{\prime}, & a b^{\prime} c^{\prime} d . \\ a^{\prime} b c^{\prime} d, & a b c^{\prime} d^{\prime}, & a^{\prime} b^{\prime} c^{\prime} d, & \\ a^{\prime} b^{\prime} c^{\prime} d^{\prime}, & a b^{\prime} c^{\prime} d^{\prime}, & a^{\prime} b c d, & \\ & a^{\prime} b c^{\prime} d^{\prime}, & a b^{\prime} c d^{\prime}, & \end{array}$

From Theorems 15 and 17, it is seen that the four regions in the first column are such that, when $x$ assumes or loses an increment from one of them, $f(x, y)$ is unchanged; they are thus like the regions $a b$ and $a^{\prime} b^{\prime}$ for functions of one variable. From Theorems 1 and 7, we see that the five regions in the second column are monotone non-decreasing and not constant; they are thus like region $a b^{\prime}$ for one variable. And from Theorems 8 and 14, we see that the five regions in the third column are monotone non-increasing and not constant, and are thus like region $a^{\prime} b$ for one variable. Of the two regions in the last column, however, we know only that $f(x, y)$ is neither monotone nondecreasing nor monotone non-increasing for changes of $x$ in them.

6. Duality considerations for monotone functions. By Theorem 1, to say that $f(x)=a x+b x^{\prime}$ is monotone non-decreasing in $(A, B)$ is equivalent to saying

$$
A<B<a+b^{\prime} .
$$

* The increment assumed, or lost, by $f(x)$ may, however, in any one of the cases, be vanishing. 
The dual of (1) is, if we represent the dual of $A$ by $A_{1}$, etc.,

$$
A_{1}>B_{1}>a_{1} b_{1}^{\prime}
$$

or

$$
A_{1}^{\prime}<B_{1}^{\prime}<a_{1}^{\prime}+b_{1} .
$$

By Theorem 8 , to say that $g(x)=a_{1} x+b_{1} x^{\prime}$ is monotone non-increasing in $\left(A_{1}^{\prime}, B_{1}^{\prime}\right)$ is equivalent to saying

$$
A_{1}^{\prime}<B_{1}^{\prime}<a_{1}^{\prime}+b_{1} .
$$

But (4) is the same as (3). Hence, the dual of " $a x+b x^{\prime}$ is monotone non-decreasing in $(A, B)$ " is " $a_{1} x+b_{1} x^{\prime}$ is monotone non-increasing in $\left(A_{1}^{\prime}, B_{1}^{\prime}\right)$."

Similar considerations apply to functions of $n$ variables.

It is now seen that Theorem 8 is the dual of Theorem 1. For, writing $a x+b x^{\prime}$ for $f(x), a$ for $f(1)$ and $b$ for $f(0)$ in Theorem 1, and applying the rule, we obtain the following proposition: A necessary and sufficient condition that $a_{1} x+b_{1} x^{\prime}$ be monotone non-increasing in $\left(A_{1}^{\prime}, B_{1}^{\prime}\right)$ is that $B_{1}>a_{1} b_{1}^{\prime}$. Substituting $a_{1}$ for $a, b_{1}$ for $b, A_{1}^{\prime}$ for $A$, and $B_{1}^{\prime}$ for $B$, we obtain: A necessary and sufficient condition that $a x+b x^{\prime}$ be monotone non-increasing in $(A, B)$ is that $B^{\prime}>a b^{\prime}$. But $B^{\prime}>a b^{\prime}$ is equivalent to $B<a^{\prime}+b$, so we have Theorem 8 .

In a similar way, it can be shown that Theorems $9,10,12,13$, and 14 are the respective duals of Theorems 2, 3, 5, 6, and 7 . Theorems 15, 16, 17, and 18 are self-dual.

7. Additive and subtractive functions. Schmidt has established the following fact concerning a Boolean function of one variable, $f(x)=a x+b x^{\prime}$ : If $\alpha<a b^{\prime}$, then $f(x+\alpha)=f(x)+\alpha$ and $f\left(x \alpha^{\prime}\right)=f(x) \alpha^{\prime} ;$ and if $\alpha<a^{\prime} b$, then $f(x+\alpha)=f(x) \alpha^{\prime}$ and $f\left(x \alpha^{\prime}\right)=f(x)+\alpha$.

I propose in this section to generalize this result to functions of $n$ variables. To this end I first lay down the following definitions.

Definition 3. A function $f\left(x_{1}, \cdots, x_{n}\right)$ is said to be additive with respect to $x_{1}$ in the domain $(A, B)$ if, for all $\alpha$ in $(A, B)$, we have

$$
\begin{aligned}
f\left(x_{1}+\alpha, x_{2}, \cdots, x_{n}\right) & =f\left(x_{1}, \cdots, x_{n}\right)+\alpha, \\
f\left(x_{1} \alpha^{\prime}, x_{2}, \cdots, x_{n}\right) & =f\left(x_{1}, \cdots, x_{n}\right) \alpha^{\prime}
\end{aligned}
$$

independent of the choice of $x_{1}, \cdots, x_{n}$. If $f\left(x_{1}, \cdots, x_{n}\right)$ is additive with respect to $x_{1}$ in the domain $(0,1)$, we say that $f\left(x_{1}, \cdots, x_{n}\right)$ is additive with respect to $x_{1}$ everywhere.

Definition 4. A function $f\left(x_{1}, \cdots, x_{n}\right)$ is said to be subtractive with respect to $x_{1}$ in the domain $(A, B)$ if, for all $\alpha$ in $(A, B)$, we have 


$$
\begin{aligned}
f\left(x_{1}+\alpha, x_{2}, \cdots, x_{n}\right) & =f\left(x_{1}, \cdots, x_{n}\right) \alpha^{\prime}, \\
f\left(x_{1} \alpha^{\prime}, x_{2}, \cdots, x_{n}\right) & =f\left(x_{1}, \cdots, x_{n}\right)+\alpha
\end{aligned}
$$

independent of the choice of $x_{1}, \cdots, x_{n}$. If $f\left(x_{1}, \cdots, x_{n}\right)$ is subtractive with respect to $x_{1}$ in the domain $(0,1)$, we say that $f\left(x_{1}, \cdots, x_{n}\right)$ is subtractive with respect to $x_{1}$ everywhere.

The following theorem includes the result, mentioned above, due to Schmidt, but is somewhat more complete, in that it shows that the given condition is necessary as well as sufficient.

THEOREM 19. A necessary and sufficient condition that a function of one variable $f(x)$ be additive in $(A, B)$ is that $B<f(1) f^{\prime}(0)$.

Proof. The condition

$$
f(x+\alpha)=f(x)+\alpha
$$

reduces to

$$
f^{\prime}(1) \alpha x+f^{\prime}(1) \alpha x^{\prime}=0,
$$

or

$$
f^{\prime}(1) \alpha=0 \text {. }
$$

The condition

$$
f\left(x \boldsymbol{\alpha}^{\prime}\right)=f(x) \boldsymbol{\alpha}^{\prime}
$$

reduces to

$$
f(0) \alpha x+f(0) \alpha x^{\prime}=0,
$$

or

$$
f(0) \alpha=0 .
$$

Equations (3) and (6) are together equivalent to

$$
\alpha\left[f^{\prime}(1)+f(0)\right]=0,
$$

or

$$
\alpha<f(1) f^{\prime}(0) .
$$

By an argument like that used in the proof of Theorem 1, it is now seen that to say that (8) holds for all $\alpha$ in $(A, B)$ is equivalent to saying that

$$
B<f(1) f^{\prime}(0) \text {. }
$$


In the above proof, it will be noticed that the condition that (2) hold for all $x$ is the same as the condition that it have a solution for some $x$; and similarly, the condition that (5) hold for all $x$ is the same as that it have a solution for some $x$. Hence, we could weaken Definition 3 (as applying to functions of one variable) to say that $f(x)$ is additive in $(A, B)$ if for all $\alpha$ in $(A, B)$ there is at least one $x$ such that

$$
f(x+\alpha)=f(x)+\alpha
$$

and at least one $x$ such that

$$
f\left(x \alpha^{\prime}\right)=f(x) \alpha^{\prime} .
$$

A similar situation will be found to obtain with respect to Definition 4 .

I next generalize Theorem 19 to functions of $n$ variables.

THEOREM 20. A necessary and sufficient condition that $f\left(x_{1}, \cdots, x_{n}\right)$ be additive with respect to $x_{1}$ in $(A, B)$ is that

$$
B<\prod_{\alpha_{i}=0,1} f\left(1, \alpha_{2}, \cdots, \alpha_{n}\right) f^{\prime}\left(0, \alpha_{2}, \cdots, \alpha_{n}\right) .
$$

Proof. For $n=1$ the theorem reduces to Theorem 19.

Suppose the theorem true for $n=k$, and let $f\left(x_{1}, \cdots, x_{k+1}\right)$ be a function of $k+1$ variables. Then, for any given value of $x_{k+1}$, as $y, f\left(x_{1}, \cdots, x_{k}, y\right)$ is a function of $k$ variables; hence, by hypothesis, a necessary and sufficient condition that $f\left(x_{1}, \cdots, x_{k}, y\right)$ be additive with respect to $x_{1}$ in $(A, B)$ is that

$$
B<\prod_{\alpha_{i}=0,1} f\left(1, \alpha_{2}, \cdots, \alpha_{k}, y\right) f^{\prime}\left(0, \alpha_{2}, \cdots, \alpha_{k}, y\right) .
$$

Hence, a necessary and sufficient condition that $f\left(x_{1}, \cdots, x_{k+1}\right)$ be additive is that (1) hold for all $y$. But to say that (1) holds for all $y$ is equivalent to saying that it holds for $y=1$ and $y=0$, hence to

$$
\begin{aligned}
& B<\prod_{\alpha_{i}=0,1} f\left(1, \alpha_{2}, \cdots, \alpha_{k}, 1\right) f^{\prime}\left(0, \alpha_{2}, \cdots, \alpha_{k}, 1\right), \\
& B<\prod_{\alpha_{i}=0,1} f\left(1, \alpha_{2}, \cdots, \alpha_{k}, 0\right) f^{\prime}\left(0, \alpha_{2}, \cdots, \alpha_{k}, 0\right) .
\end{aligned}
$$

These two inclusions are equivalent to the single inclusion

$$
B<\prod_{\alpha_{i}=0,1} f\left(1, \alpha_{2}, \cdots, \alpha_{k+1}\right) f^{\prime}\left(0, \alpha_{2}, \cdots, \alpha_{k+1}\right) .
$$

Hence, the theorem holds for $n=k+1$ if it holds for $n=k$.

THEOREM 21. A necessary and sufficient condition that $f\left(x_{1}, \cdots, x_{n}\right)$ be additive with respect to $x_{1}$ everywhere is that

$$
f\left(1, \alpha_{2}, \cdots, \alpha_{n}\right)=1, \quad f\left(0, \alpha_{2}, \cdots, \alpha_{n}\right)=0 \quad\left[\alpha_{i}=0,1\right] .
$$


The theorem follows from Theorem 20.

THEOREM 22. The only function that is additive with respect to $x_{1}$ everywhere is

$$
f\left(x_{1}, \cdots, x_{n}\right)=x_{1} .
$$

Proof. Substituting the values given by Theorem 21 into the identity

$$
f\left(x_{1}, \cdots, x_{n}\right)=f(1, \cdots, 1) x_{1} \cdots x_{n}+\cdots+f(0, \cdots, 0) x_{1}^{\prime} \cdots x_{n}^{\prime},
$$

we find

$$
\begin{aligned}
f\left(x_{1}, \cdots, x_{n}\right) & =x_{1} \cdots x_{n}+\cdots+x_{1} x_{2}^{\prime} \cdots x_{n}^{\prime} \\
& =x_{1}\left(x_{2} \cdots x_{n}+\cdots+x_{2}^{\prime} \cdots x_{n}^{\prime}\right) \\
& =x_{1} .
\end{aligned}
$$

The following theorems on subtractive functions are stated without proof, since the proofs are analogous to the corresponding proofs for additive functions.

TheOREM 23. A necessary and sufficient condition that a function of one variable $f(x)$ be subtractive in $(A, B)$ is that

$$
B<f^{\prime}(1) f(0) \text {. }
$$

THEOREM 24. A necessary and sufficient condition that $f\left(x_{1}, \cdots, x_{n}\right)$ be subtractive with respect to $x_{1}$ in $(A, B)$ is that

$$
B<\prod_{\alpha_{i}=0,1} f^{\prime}\left(1, \alpha_{2}, \cdots, \alpha_{n}\right) f\left(0, \alpha_{2}, \cdots, \alpha_{n}\right) .
$$

THEOREM 25. A necessary and sufficient condition that $f\left(x_{1}, \cdots, x_{n}\right)$ be subtractive with respect to $x_{1}$ everywhere is that

$$
f\left(1, \alpha_{2}, \cdots, \alpha_{n}\right)=0, \quad f\left(0, \alpha_{2}, \cdots, \alpha_{n}\right)=1 \quad\left[\alpha_{i}=0,1\right] .
$$

THEOREM 26. The only function that is subtractive with respect to $x_{1}$ everywhere is

$$
f\left(x_{1}, \cdots, x_{n}\right)=x_{1}^{\prime} .
$$

8. Remarks on additive and subtractive functions. It will doubtless have been noticed that, for a function of one variable, the region, $a b^{\prime}$, in which $f(x)$ is monotone non-decreasing and not constant, coincides with the region in which $f(x)$ is additive. Such is not the case, however, for functions of two or more variables. Thus, for example, for a function of two variables, $f(x, y)=a x y+b x y^{\prime}+c x^{\prime} y+d x^{\prime} y^{\prime}, f(x, y)$ is monotone non-decreasing with respect to $x$ and not constant in the five regions $a b c^{\prime} d, a b c d^{\prime}, a b c^{\prime} d^{\prime}, a b^{\prime} c^{\prime} d^{\prime}$, 
$a^{\prime} b c^{\prime} d$, while the only region in which $f(x, y)$ is additive with respect to $x$ is $a b c^{\prime} d^{\prime}$. It is also of some interest to observe that a function of $n$ variables is never additive in the same domain with respect to two or more variables.

Analogous remarks apply to subtractive domains.

9. Duality considerations. By an argument similar to that used in $\S 6$, it can be shown that the dual of " $a x+b x^{\prime}$ is additive in $(A, B)$ " is " $a_{1} x+b_{1} x^{\prime}$ is subtractive in $\left(A_{1}^{\prime}, B_{1}^{\prime}\right)$." Thus, Theorems $23,24,25$, and 26 are the duals, respectively, of Theorems $19,20,21$, and 22 .

10. Continuity. In ordinary analysis, we say that a function $f(x)$ is uniformly continuous if for every $\epsilon>0$ there is a $\delta>0$ such that

$$
\text { if }\{|x-y|<\delta\} \text { then }\{|f(x)-f(y)|<\epsilon\} .
$$

It is of some interest to try to extend the notion of "uniform continuity" to Boolean algebra.

To make such an extension it is first necessary to find in Boolean algebra some analogue of the operation $|x-y|$. Probably the best such analogue* is $x \circ y$. From the usual geometrical representation of $x \circ y$ it is seen that $x \circ y$ is the total amount by which $x$ and $y$ fail to coincide; if $x \circ y=0$, then $x=y$.

On the basis of this analogy, we may lay down the following definition.

Definition 5. A function $f(x)$ of a Boolean variable is said to be uniformly continuous if, for every Boolean element $\epsilon \neq 0$, there exists an element $\delta \neq 0$ such that

$$
\text { if }\{x \circ y<\delta\} \text { then }\{f(x) \circ f(y)<\epsilon\} .
$$

The definition can be extended to functions of $n$ variables as follows.

Definition 6. A function $f\left(x_{1}, \cdots, x_{n}\right)$ of $n$ Boolean variables is said to be uniformly continuous if, for every Boolean element $\epsilon \neq 0$, there exists an ordered set of Boolean elements $\delta_{1}, \cdots, \delta_{n}$, with $\delta_{i} \neq 0$, such that if $\left\{\left(x_{1} \circ y_{1}<\delta_{1}\right), \cdots,\left(x_{n} \circ y_{n}<\delta_{n}\right)\right\}$ then $\left\{f\left(x_{1}, \cdots, x_{n}\right) \circ f\left(y_{1}, \cdots, y_{n}\right)<\epsilon\right\}$.

The concept just defined, however, despite the fact that it is analogous to one of the dominant concepts of ordinary analysis, is not a very important one in connection with Boolean functions, for, as shown in the following theorems, every Boolean function is uniformly continuous.

THEOREM 27. If $f\left(x_{1}, \cdots, x_{n}\right)$ is any Boolean function, then

$$
f\left(x_{1}, \cdots, x_{n}\right) \circ f\left(y_{1}, \cdots, y_{n}\right)<\left(x_{1} \circ y_{1}\right)+\cdots+\left(x_{n} \circ y_{n}\right) .
$$

* For a treatment of $x \circ y$ as an analogue of $|x-y|$ see P. J. Daniell, The modular difference of classes, Bulletin of the American Mathematical Society, vol. 23 (1917), pp. 446-450. For a treatment of $x \circ y$ as an analogue of "distance," see M. H. Stone, Postulates for Boolean algebras and generalized Boolean algebra, American Journal of Mathematics, vol. 57 (1935), pp. 703-732. 
Proof. It is easily seen that when the expression $f\left(x_{1}, \cdots, x_{n}\right) \circ f\left(y_{1}, \cdots, y_{n}\right)$ is expanded, all terms where $x_{i}$ and $y_{i}$ are primed or not primed together (such as $x_{1} \cdots x_{n} \cdot y_{1} \cdots y_{n}$ or $\left.x_{1}^{\prime} \cdots x_{n-1}^{\prime} \cdot x_{n} \cdot y_{1}^{\prime} \cdots y_{n-1}^{\prime} \cdot y_{n}\right)$ have discriminants $=0$. On the other hand, if we expand the expression $\left(x_{1} \circ y_{1}\right)+\cdots+\left(x_{n} \circ y_{n}\right)$, we see that the expansion will be as follows: All terms where $x_{i}$ and $y_{i}$ are primed or not primed together have discriminants $=0$, and all other terms have discriminants $=1$. Hence the theorem is proved.

THEOREM 28. Every Boolean function is uniformly continuous.

Proof. Let $\epsilon \neq 0$ be arbitrary, and choose $\delta_{1}=\cdots=\delta_{n}=\epsilon$. Then, by Theorem 25, when

$$
\left(x_{1} \circ y_{1}<\delta_{1}\right), \cdots,\left(x_{n} \circ y_{n}<\delta_{n}\right),
$$

we have

$$
\begin{aligned}
f\left(x_{1}, \cdots, x_{n}\right) \circ f\left(y_{1}, \cdots, y_{n}\right) & <\left(x_{1} \circ y_{1}\right)+\cdots+\left(x_{n} \circ y_{n}\right) \\
& <\delta_{1}+\cdots+\delta_{n}=\epsilon+\cdots+\epsilon=\epsilon .
\end{aligned}
$$

11. Remarks on continuity. Since we have now seen that all Boolean functions are uniformly continuous, it would be interesting to inquire what non-Boolean functions, if any, are uniformly continuous. Such an investigation, however, would lie outside the bounds I have set myself. I shall merely remark here that one can construct examples to show that some, but not all non-Boolean functions are uniformly continuous. From this it seems likely that the continuity concept might be of importance in the study of a more general type of functions. The relation of Boolean functions to uniformly continuous functions in Boolean algebra is somewhat similar to the relation of polynomials to uniformly continuous functions in ordinary algebra.

12. One-valued inverses of functions. When $z$ is a Boolean function of $x$, then it is possible to consider $x$ as a function of $z$, say $g(z)$, where $g(z)$, however, is not, in general, one-valued. Similarly, if $z$ is a function of $x_{1}, \cdots, x_{n}$, then, again, we may consider $x_{1}$ as a function of $z, x_{2}, \cdots, x_{n}$ where $g$, again, may be many-valued.

It is of some interest to investigate the conditions on the given function in order that the inverse function be single-valued. More generally, it is of interest to find, for any $f$, what domains are such that for $x$ in them $f$ has a single-valued inverse. This question has been solved by Schmidt for a special case: namely, he has shown that a Boolean function of one variable, $f(x)=a x+b x^{\prime}$, has a one-valued inverse for $x$ in $(a b, a+b)$ and also for $x$ in $(0, a \circ b)$. I propose to find all the domains for a function of $n$ variables. I first lay down the following formal definition. 
Definition 7. A Boolean function $f\left(x_{1}, \cdots, x_{n}\right)$ is said to have a onevalued inverse with respect to $x_{1}$ in the domain $(A, B)$ if, for every $u$ and $v$ in $(A, B)$, we have

$$
\left[f\left(u, x_{2}, \cdots, x_{n}\right)=f\left(v, x_{2}, \cdots, x_{n}\right)\right] \supset(u=v),
$$

independent of $x_{2}, \cdots, x_{n}$. If $f\left(x_{1}, \cdots, x_{n}\right)$ has a one-valued inverse for $x_{1}$ in the domain $(0,1)$, we say that $f\left(x_{1}, \cdots, x_{n}\right)$ has a one-valued inverse with respect to $x_{1}$ everywhere.

Preliminary to establishing the principal theorem of this section, it is convenient to prove the following lemma.

LEMMA TO THEOREM 29. If every solution of $a\left(v_{1} \circ v_{2}\right)=0$ is also a solution of $b\left(v_{1} \circ v_{2}\right)=0$, then $b<a$.

Proof. Suppose that every solution of

$$
a\left(v_{1} \circ v_{2}\right)=0
$$

is also a solution of

$$
b\left(v_{1} \circ v_{2}\right)=0 .
$$

A particular solution of (1) is $v_{1}=a, v_{2}=1$, since $a(a \circ 1)=a a^{\prime}=0$. Hence, this must also be a solution of (2); hence $b(a \circ 1)=b a^{\prime}=0$. Hence $b<a$.

THEOREM 29. A function $f(x)=a x+b x^{\prime}$ has a one-valued inverse in those and only those domains that are of the form

$$
(A, A+B[a \circ b]) \text {. }
$$

Proof. First suppose that $y_{1}$ and $y_{2}$ lie in a domain $(A, A+B[a \circ b])$. Then there exists a $v_{1}$ and a $v_{2}$ such that

$$
y_{1}=A v_{1}^{\prime}+(A+B[a \circ b]) v_{1}, \quad y_{2}=A v_{2}^{\prime}+(A+B[a \circ b]) v_{2} .
$$

From (1), we see that

$$
y_{1} \circ y_{2}=A^{\prime} B(a \circ b)\left(v_{1} \circ v_{2}\right) \text {. }
$$

Now,

$$
f\left(y_{1}\right) \circ f\left(y_{2}\right)=(a \circ b)\left(y_{1} \circ y_{2}\right) .
$$

Thus, from (2) and (3),

$$
f\left(y_{1}\right) \circ f\left(y_{2}\right)=y_{1} \circ y_{2} .
$$

Hence, if $f\left(y_{1}\right)=f\left(y_{2}\right)$, then $y_{1}=y_{2}$; hence $f(x)$ has a one-valued inverse in $(A, A+B[a \circ b])$. 
Suppose, on the other hand, that the domain $(A, A+B)$ is such that within it $f(x)$ has a one-valued inverse. Let $y_{1}$ and $y_{2}$ be any elements in $(A, A+B)$; then there exists a $v_{1}$ and a $v_{2}$ such that

$$
y_{1}=A v_{1}^{\prime}+(A+B) v_{1}, \quad y_{2}=A v_{2}^{\prime}+(A+B) v_{2} .
$$

By hypothesis,

$$
\left[f\left(y_{1}\right)=f\left(y_{2}\right)\right] \supset\left(y_{1}=y_{2}^{\prime}\right),
$$

which is equivalent to

$$
\left[(a \circ b)\left(y_{1} \circ y_{2}\right)=0\right] \supset\left[y_{1} \circ y_{2}=0\right] .
$$

Substituting (5) in (7) and simplifying, we have

$$
\left[(a \circ b) A^{\prime} B\left(v_{1} \circ v_{2}\right)=0\right] \supset\left[A^{\prime} B\left(v_{1} \circ v_{2}\right)=0\right] .
$$

Applying the lemma to (8), we have

$$
A^{\prime} B<A^{\prime} B(a \circ b) \text {. }
$$

But (9) is equivalent to

$$
B=A B+B(a \circ b) .
$$

Thus

$$
(A, A+B)=(A, A+A B+B[a \circ b])=(A, A+B[a \circ b]) .
$$

It will be noticed that the two results previously mentioned as having been given by Schmidt are special cases of Theorem 29. To show that $f(x)$ has a one-valued inverse in $(a b, a+b)$, set $A=a b$ and $B=1$. To show that $f(x)$ has a one-valued inverse in $(0, a \circ b)$, set $A=0$ and $B=1$. It is evident that the most inclusive domains are got by taking $B=1$.

I now generalize Theorem 29 to functions of $n$ variables.

THEOREM 30. A function $f\left(x_{1}, \cdots, x_{n}\right)$ has a one-valued inverse with respect to $x_{1}$ in those and only those domains that are of the form

$$
\left(A, A+B \prod_{\alpha_{i}=0,1}\left[f\left(1, \alpha_{2}, \cdots, \alpha_{n}\right) \circ f\left(0, \alpha_{2}, \cdots, \alpha_{n}\right)\right]\right) .
$$

Proof. For $n=1$, we have Theorem 29.

Suppose the theorem true for $n=k$. Let $f\left(x_{1}, \cdots, x_{k}, x_{k+1}\right)$ be any function of $k+1$ variables. Then for any fixed value of $x_{k+1}$, as $y, f\left(x_{1}, \cdots, x_{k}, y\right)$ is a function of $k$ variables. Hence by the induction hypothesis we have: $f\left(x_{1}, \cdots, x_{k}, y\right)$ has a one-valued inverse with respect to $x_{1}$ in the domain $(A, A+C)$ if and only if there is a $B$ such that 


$$
A+C=A+B \prod_{\alpha_{i}=0,1}\left[f\left(1, \alpha_{2}, \cdots, \alpha_{k}, y\right) \circ f\left(0, \alpha_{2}, \cdots, \alpha_{k}, y\right)\right] .
$$

But there is a $B$ such that (1) holds if and only if

$$
C<A+\prod_{\alpha_{i}=0,1}\left[f\left(1, \alpha_{2}, \cdots, \alpha_{k}, y\right) \circ f\left(0, \alpha_{2}, \cdots, \alpha_{k}, y\right)\right] .
$$

Thus, a necessary and sufficient condition that $f\left(x_{1}, \cdots, x_{k}, y\right)$ have a onevalued inverse with respect to $x_{1}$ in $(A, A+C)$ is that (2) hold. Hence, a necessary and sufficient condition that $f\left(x_{1}, \cdots, x_{k}, x_{k+1}\right)$ have a one-valued inverse with respect to $x_{1}$ in $(A, A+C)$ is that (2) hold for all $y$. But to say that (2) holds for all $y$ is equivalent to saying it holds for $y=1$ and $y=0$, hence equivalent to

$$
\begin{aligned}
& C<A+\prod_{\alpha_{i}=0,1}\left[f\left(1, \alpha_{2}, \cdots, \alpha_{k}, 1\right) \circ f\left(0, \alpha_{2}, \cdots, \alpha_{k}, 1\right)\right], \\
& C<A+\prod_{\alpha_{i}=0,1}\left[f\left(1, \alpha_{2}, \cdots, \alpha_{k}, 0\right) \circ f\left(0, \alpha_{2}, \cdots, \alpha_{k}, 0\right)\right],
\end{aligned}
$$

which is equivalent to

$$
C<A+\prod_{\alpha_{i}=0,1}\left[f\left(1, \alpha_{2}, \cdots, \alpha_{k+1}\right) \circ f\left(0, \alpha_{2}, \cdots, \alpha_{k+1}\right)\right] .
$$

But to say that (4) holds, is equivalent to saying that there exists a $B$ such that

$$
A+C=A+B \prod_{\alpha_{i}=0,1}\left[f\left(1, \alpha_{2}, \cdots, \alpha_{k+1}\right) \circ f\left(0, \alpha_{2}, \cdots, \alpha_{k+1}\right)\right] .
$$

Hence, the theorem holds for $n=k+1$ if it holds for $n=k$.

THEOREM 31. A necessary and sufficient condition that a function $f\left(x_{1}, \cdots, x_{n}\right)$ have a one-valued inverse with respect to $x_{1}$ everywhere is that

$$
f\left(1, \alpha_{2}, \cdots, \alpha_{n}\right)=f^{\prime}\left(0, \alpha_{2}, \cdots, \alpha_{n}\right) \quad\left[\alpha_{i}=0,1\right] .
$$

Proof. Since every domain in which $f\left(x_{1}, \cdots, x_{n}\right)$ has a one-valued inverse with respect to $x_{1}$ is, by Theorem 30 , of the form

$$
\left(A, A+B \prod_{\alpha_{i}=0,1}\left[f\left(1, \alpha_{2}, \cdots, \alpha_{n}\right) \circ f\left(0, \alpha_{2}, \cdots, \alpha_{n}\right)\right]\right),
$$

it is clear that if $f\left(x_{1}, \cdots, x_{n}\right)$ has a one-valued inverse with respect to $x_{1}$ in the domain $(0,1)$, we must take $A=0$ and

$$
A+B \prod_{\alpha_{\imath}=0,1}\left[f\left(1, \alpha_{2}, \cdots, \alpha_{n}\right) \circ f\left(0, \alpha_{2}, \cdots, \alpha_{n}\right)\right]=1 .
$$

Hence, 


$$
f\left(1, \alpha_{2}, \cdots, \alpha_{n}\right) \circ f\left(0, \alpha_{2}, \cdots, \alpha_{n}\right)=1 \quad\left[\alpha_{i}=0,1\right] .
$$

Hence,

$$
f\left(1, \alpha_{2}, \cdots, \alpha_{n}\right)=f^{\prime}\left(0, \alpha_{2}, \cdots, \alpha_{n}\right) \quad\left[\alpha_{i}=0,1\right] .
$$

THEOREM 32. Those and only those functions have one-valued inverses with respect to $x_{1}$ everywhere that are of the form

$$
\begin{aligned}
f\left(x_{1}, \cdots, x_{n}\right)= & {\left[x_{1} \Delta f(1,1, \cdots, 1)\right] x_{2} \cdots x_{n}+\cdots } \\
& +\left[x_{1} \Delta f(1,0, \cdots, 0)\right] x_{2}^{\prime} \cdots x_{n}^{\prime} .
\end{aligned}
$$

This theorem is an immediate consequence of Theorem 31 .

The next theorem tells us the form which inverse functions have when they exist.

THEOREM 33. If $f\left(x_{1}, \cdots, x_{n}\right)=z$ has a one-valued inverse with respect to $x_{1}$ everywhere, this inverse function with respect to $x_{1}$ is

$$
x_{1}=f\left(z, x_{2}, \cdots, x_{n}\right) \text {. }
$$

Proof. Under the given hypothesis, we have, by Theorem 31,

$$
\begin{aligned}
& f(1,1, \cdots, 1) x_{1} \cdots x_{n}+\cdots+f(1,0, \cdots, 0) x_{1} \cdot x_{2}^{\prime} \cdots x_{n}^{\prime} \\
& \quad+f^{\prime}(1,1, \cdots, 1) x_{1}^{\prime} \cdot x_{2} \cdots x_{n}+\cdots+f^{\prime}(1,0, \cdots, 0) x_{1}^{\prime} \cdots x_{n}^{\prime}=z,
\end{aligned}
$$

which is equivalent to

$$
\begin{aligned}
{[z \circ f(1,1, \cdots, 1)] x_{1} \cdots } & x_{n}+\cdots+[z \circ f(1,0, \cdots, 0)] x_{1} \cdot x_{2}^{\prime} \cdots x_{n}^{\prime} \\
+ & {\left[z \circ f^{\prime}(1,1, \cdots, 1)\right] x_{1}^{\prime} \cdot x_{2} \cdots x_{n}+\cdots } \\
+ & {\left[z \circ f^{\prime}(1,0, \cdots, 0)\right] x_{1}^{\prime} \cdots x_{n}^{\prime}=0 }
\end{aligned}
$$

or, again, to

$$
\begin{aligned}
\left\{[z \circ f(1,1, \cdots, 1)] x_{2} \cdots x_{n}\right. & +\cdots \\
& \left.+[z \circ f(1,0, \cdots, 0)] x_{2}^{\prime} \cdots x_{n}^{\prime}\right\} \Delta x_{1}=0 .
\end{aligned}
$$

Equation (3) has the unique solution

$$
\begin{aligned}
x_{1} & =[z \Delta f(1,1, \cdots, 1)] x_{2} \cdots x_{n}+\cdots+[z \Delta f(1,0, \cdots, 0)] x_{2}^{\prime} \cdots x_{n}^{\prime} \\
& =f\left(z, x_{2}, \cdots, x_{n}\right),
\end{aligned}
$$

as was to be shown.

The University of California, Berkeley, Calif. 